\title{
EQUADIFF 4
}

\section{Siegfried Dümmel}

On some inverse problems for partial differential equations

In: Jiří Fábera (ed.): Equadiff IV, Czechoslovak Conference on Differential Equations and Their Applications. Proceedings, Prague, August 22-26, 1977. Springer-Verlag, Berlin, 1979. Lecture Notes in Mathematics, 703. pp. [93]--98.

Persistent URL: http://dml.cz/dmlcz/702208

\section{Terms of use:}

(C) Springer-Verlag, 1979

Institute of Mathematics of the Academy of Sciences of the Czech Republic provides access to digitized documents strictly for personal use. Each copy of any part of this document must contain these Terms of use.

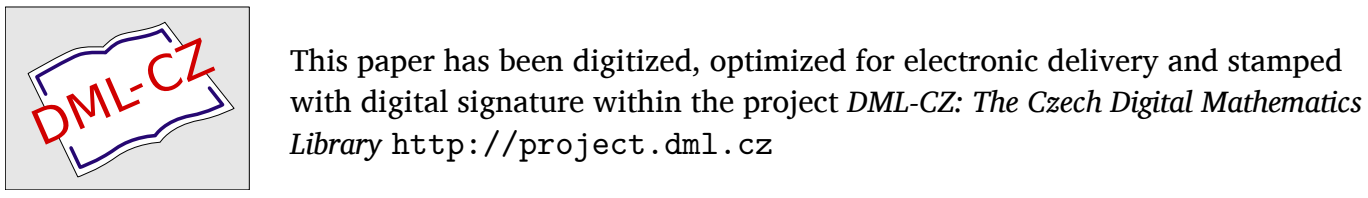


ON SOME INVERSE PROBLEMS FOR PARTIAL

DIFFEIISNTIAL EQUATIONS

S. Dümmel, Karl-Marx-stadt

\section{Introduction}

By an inverse problem for a differential equation we understand any problem in which the coefficients or the right-hand side of the differential equation are to be determined from some information on the solutions of this equation. We confine ourselves to two special cases of second order linear parabolic equations. For hyperbolic equations we refer to the book of V. T. Romanov [15].

Let $u$ be a solution of a Cauchy problem or of an initial-boundary value problem. We shall investigate the question what further information on $u$ is sufficient for the uniqueness of the unknown coefficient. For the case that the right hand side of the parabolic equation is unknown such investigations can be found $e . g$. in the following papers: W. T. Ivanov, G. P. Smirnov, F. W. Lubyšev [7], A. Fasano [4], W. M. Isakov [5] and in the book Lavrentiev, Romanov, Vasiliev [10].

The case that the unknown function is the coefficient at $u$ in the parabolic equation is considered in several papers of M. M. Lavrentiev and K. G. Resnizkaja ([8], [9], [12], [13], [14]). These authors

assumed that the unknown coefficient is only a function of one space variable. Coefficients of several variables are considered $e$. g. by A. D. Iskenderov [6] and I. Ja. Besnookenko [1], where the unknown coefficients are functions of $n-1$ space variables and of the time and $u$ is a function of $n$ space variables and of the time.

In our lecture we shall consider the question of uniqueness for the parabolic equations

and

$$
u_{t}(x, t)-q(x) \Delta u(x, t)=0
$$

$$
u_{t}(x, t)-\sum_{i=1}^{n} \frac{\partial}{\partial x_{i}}\left(p(x) u_{x_{i}}(x, t)\right)=0
$$

with $x=\left(x_{1}, x_{2}, \ldots, x_{n}\right) \quad(n=1,2,3, \ldots)$, where $q(x)$ and $p(x)$ are unknown. For the equation (1.2) there exist some papers by $G$. Chavent ([2], [3]) who determines $\mathrm{p}$ by a gradient method.

We use the following notations: $R^{n}$ is the $n$-dimensional Euclidean space $(n=1,2,3, \ldots), x=\left(x_{1}, x_{2}, \ldots, x_{n}\right) \in R^{n}, D$ a bounded region of $R^{n}$ with a sufficiently smooth boundary $a D$. For $T>0$ we define $Z_{T}=$ $=\mathrm{D} \times(0, \mathrm{~T}), \Gamma_{\mathrm{T}}=\mathrm{D} \times[0, \mathrm{~T}]$. 
2. Uniqueness theorems with additional conditions on $u$ for a fixed time

We consider the following initial-boundary value problem

$$
\begin{aligned}
& u_{t}(x, t)-q(x) \Delta u(x, t)=0 \text { for }(x, t) \in Z_{T}, \\
& u(x, 0)=\varphi(x) \quad \text { for } x \in D, \\
& u(x, t)=\psi(x, t) \text { for }(x, t) \in \Gamma_{T},
\end{aligned}
$$

where $q \in C(\bar{D}), q(x)>0$ for all $x \in \bar{D}, u \in C^{2}\left(\bar{z}_{T}\right)$,

$u \in C^{3}\left(z_{T}\right),\left|\Delta u_{t}(x, t)\right| \leqq K$ for all $(x, t) \in z_{T}$,

$\varphi \in C^{2}(\bar{D}), \Delta \varphi(x)=0$ for all $x \in \bar{D}, \psi \in G\left(\Gamma_{T}\right)$,

$\psi_{t} \in C\left(\Gamma_{T}\right), \varphi(x)=\psi(x, 0)$ for all $x \in \partial D$.

If $q$ is known and $u$ is a solution of the problem (2.1) (2.2) (2.3), then $u$ is unique. Now let $q$ be unknown. Then for the uniqueness of $q$ in addition to (2.1) (2.2) (2.3) we need a further information on $u$. We demand that for a fixed $t_{1}$ with $0<t_{1}<T$ there is a function $h$ with $h \in C^{2}(\bar{D}),|\Delta h(x)| \geqq t_{1}^{\alpha}\left(0<\alpha<\frac{1}{2}\right)$ for all $x \in \bar{D}$ such that

$$
u\left(x, t_{1}\right)=h(x) \quad \text { for } x \in \bar{D} \text {. }
$$

Then we obtain

Theorem 1. If $\varphi, \psi, h$ are given functions with the above properties, if $(q, u)$ and $(\bar{q}, \bar{u})$ are two pairs of functions satisfying $(2.1)-(2.4)$ and if

$$
0<t_{1}<\left(\frac{2 \lambda_{1}}{k}\right)^{\frac{1}{1-2 \alpha}},
$$

where $K$ and $\alpha$ have been introduced above and $\lambda_{1}$ is the smallest eigenvalue of the Dirichlet problem in $D$ for the elliptic operator $q \Delta u$, then $q=\bar{q}$ and $u=\bar{u}$.

Proof. Let $(q, u)$ and $(\bar{q}, \bar{u})$ be two pairs of functions satisfying $(2.1)-(2.4)$. We introduce the notation $w=u_{t}, \bar{w}=\bar{u}_{t}, \quad \tilde{w}=$ $w-\bar{w}, \tilde{q}=q-\bar{q}$. Then it can be shown that

$$
\begin{array}{ll}
\tilde{w}_{t}-q \Delta \tilde{w}=\tilde{q} \Delta \bar{w} & \text { in } z_{T}, \\
\tilde{w}^{t}(x, 0)=0 & \text { on } \bar{D}, \\
\tilde{w}(x, t)=0 & \text { on } \Gamma_{T} .
\end{array}
$$

By Fourier's separation method $\tilde{w}$ can be represented in the form

$$
\tilde{w}(x, t)=\sum_{k=1}^{\infty} \int_{0}^{t} \int_{D} \tilde{q}(y) \Delta w(y, \tau) g_{k}(y) \text { dye } e_{k}^{-\lambda_{k}(t-\tau)} d \tau g_{k}(y)
$$

where $\left\{g_{k}\right\}$ is a complete orthogonal system (in $I^{2}(D)$ ) of corresponding eigenfunctions and $\left\{\lambda_{k}\right\}$ the system of corresponding eigenvalues. If we denote the norm in $I^{2}(D)$ by $\|\cdot\|$, then we can show that

$$
\|\widetilde{w}(x, t)\|^{2} \leqq \frac{1}{2 \lambda_{1}} \int_{0}^{t} \int_{D}(\widetilde{q}(y) \Delta \bar{w}(y, \tau))^{2} d y d \tau \leqq \frac{K}{2 \lambda_{1}} t\left\|_{q}\right\|^{2} .
$$


For $t=t_{1}$ we obtain

$$
\tilde{w}\left(x, t_{1}\right)=\tilde{q}(x) \Delta h(x) \text {. }
$$

Hence

$$
\|\widetilde{q}\|^{2} \leqq \frac{1}{t_{1}^{2 \alpha}}\|\widetilde{q} \Delta h\|^{2} \leqq \frac{K}{2 \lambda_{1}} t_{1}^{1-2 \alpha}\left\|_{q}\right\|^{2} \text {. }
$$

By (2.5) we have $\frac{K}{2 \lambda_{1}} t_{1}^{1-2 a}<1$ and thus $\|\tilde{q}\|=0$. This completes the proof of the theorem.

The method of the proof of Theorem 1 can also be used in the case of the equation (1.2) if $n=1$. Thus now let $n=1$. We consider

$$
\text { (2.6) } \quad u_{t}(x, t)-\frac{\partial}{\partial x}\left(p(x) u_{x}(x, t)\right)=0 \quad \text { for }(x, t) \in z_{T}
$$

with the initial condition (2.2) and the boundary condition (2.3). $u$ and $\psi$ shall satisfy the same hypotheses as before. Furthermore we suppose that $D=(a, b), \varphi$ is constant, $p \in C^{1}([a, b]), p(x)>0$ for all $x \in[a, b]$ and $(2.4)$ holds with $h \in C^{2}([a, b]),\left|h^{\prime}(x)\right| \geqq t_{1}^{\alpha} \quad\left(0<\alpha<\frac{1}{4}\right)$ for all $x \in[a, b]$. Finally we demand that $p(a)$ is known:

$$
\text { (2.7) } \quad p(a)=c \text {. }
$$

Now we obtain a theorem analogous to Theorem 1, where we omit the exact bound for $t_{1}$.

Theorem 2. If $\varphi, \psi, h$ are given functions with the above properties, if $(\mathrm{p}, \mathrm{u})$ and $(\overline{\mathrm{p}}, \overline{\mathrm{u}})$ are two pairs of functions satisfying $(2.6),(2.2)-(2.4),(2.7)$ and if $t_{1}$ is sufficiently small, then $\mathrm{p}=\overline{\mathrm{p}}$ and $\mathrm{u}=\overline{\mathrm{u}}$.

Proof. Using the analogous notation and the same method as in the proof of Theorem 1 we obtain

$$
\|\tilde{w}(x, t)\|^{2}=M t(\|\tilde{p}\|+\|\tilde{p} \cdot\|)^{2},
$$

where $M$ is a constant and $\tilde{p}^{\prime}=\frac{d \tilde{p}}{d x}$.

For $t=t_{1}$ we have

Hence

and

$$
\tilde{w}\left(x, t_{1}\right)-\frac{d}{d x}(\tilde{p}(x) h \cdot(x))=0 .
$$

$$
\tilde{p}(x)=\frac{1}{h^{\prime}(x)} \int_{a}^{x} \tilde{w}\left(\xi, t_{1}\right)
$$

Using (2.8) and (2.9) one can estimate $\|\tilde{p}\|$ and $\|\tilde{p} \cdot\|$ by $\left\|\tilde{r}\left(x, t_{1}\right)\right\|$. Then by an analogous conclusion as in the proof of Theorem 1 we obtain the assertion of Theorem 2.

\section{Uniqueness theorems for the case that $u(x, t)$ is analytic in $t$}

Now we shall use another method for proving the uniqueness of $p(x)$ in (2.1). This proof is due to H. P. Iinke. For simplicity we 
formulate the following considerations for the case $n=1$. But it is also possible to treat the case of several variables in a similar manner.

Thus let $\mathrm{n}=1$ and $\mathrm{Z}_{\infty}=\mathrm{R}^{1} \times(0, \infty)$. We consider the following Cauchy problem:

$$
\begin{aligned}
& u_{t}(x, t)-\frac{\partial}{\partial x}\left(p(x) u_{x}(x, t)\right)=0 \text { for }(x, t) \in Z_{\infty} \\
& u(x, 0)=\varphi(x) \text { for } x \in R^{1},
\end{aligned}
$$

where $p(x)=\sum_{k=0}^{\infty} p_{k} x^{k}, p(x)>0$ for all $x \in R^{1}$ and $\varphi \in C^{\infty}\left(R^{1}\right)$. Let $0>0$ and

$$
p(0)=p_{0}=c
$$

Furthermore we assume that $u, u_{x}, u_{x x}$ are representable in the form

$$
\begin{aligned}
& u(x, t)=\sum_{k=0}^{\infty} u_{k}(x) t^{k}, u_{x}(x, t)=\sum_{k=0}^{\infty} u_{k}^{\prime}(x) t^{k}, \\
& u_{x x}(x, t)=\sum_{k=0}^{\infty} u_{k}^{\prime \prime}(x) t^{k}
\end{aligned}
$$

for $(x, t) \in \bar{z}_{\infty}$, where $u_{k} \in C^{2}\left(R^{1}\right)$ and $u_{k}$, $u_{k}^{\prime \prime}$ are the derivatives of $u_{k}$. Finally we suppose that there is a function $g \in C^{\infty}([0, \infty))$ such that

$$
u(0, t)=g(t) \text { for } 0 \leqq t \text {. }
$$

Then the following theorem holds.

Theorem 3. Let $\varphi$ and $g$ be given functions and $p$ and $u$ unknown functions with the above properties and $c$ a given positive number. If in addition $p_{2 \nu}=0$ for $\nu=1,2,3, \ldots$ and $\varphi^{\prime}(0)=0$, then there exists at most one $p$ such that (3.1) - (3.4) are fulfilled.

Proof. From (3.1) we obtain

and

$$
\sum_{k=0}^{\infty}(k+1) u_{k+1}(x) t^{k}=\sum_{k=0}^{\infty} \frac{d}{d x}\left(p(x) u_{k}(x)\right) t^{k}
$$

with

$$
k u_{k}(x)=\frac{d}{d x}\left(p(x) u_{k-1}^{\prime}(x)\right) \quad(k=1,2,3, \ldots)
$$

$$
u_{0}(x)=\varphi(x)
$$

Since by (3.4) $u(0, t)$ is known, also all $u_{k}(0)$ are known. Using the equality

$$
p_{k}=\frac{p^{k}(0)}{k !}
$$

and the additional assumptions of the theorem, one can show that

$$
p_{2 v-1}=F_{v}\left(u(0), p_{0}, p_{1}, \ldots, p_{2 v-2}, \varphi^{\prime}(0), \ldots, \varphi^{(2 v)}(0)\right),
$$

where the $F_{p}$ are known functions and $\nu=1,2,3, \ldots$. Thus all $p_{k}$ are uniquely determined by the given conditions and the theorem is proved.

It is also possible to prove an analogous theorem for the case that $p_{2 p-1}=0$ for all $p=1,2,3, \ldots$. 
4. Reduction of a one-dimensional problem to an inverse Sturm-Liouville problem

At last we consider the following one-dimensional problem:

$$
\begin{array}{ll}
\text { (4.1) } & u_{t}(x, t)-\frac{\partial}{\partial x}\left(p(x) u_{x}(x, t)\right)=0 \quad \text { for } a<x<b, 0<t, \\
(4.2) & u(x, 0)=\delta(x-a) \text { for } a \leqq x \leqq b, \\
(4.3) & u_{x}(a, t)-h u(a, t)=0 \quad \text { for } 0<t,
\end{array}
$$$$
\text { (4.3) } \quad u_{x}(a, t)-h u(a, t)=0 \quad \text { for } 0<t \text {, }
$$

where $S_{\text {is }}$ the Dirac delta function, $h$ and $H$ are real numbers, $p(x)$ $>0$ for all $x \in[a, b], p \in c^{2}(a, b)$ and $u$ is a (generalized) solution of (4.1) - (4.3). Furthernore we suppose that

$$
p(a)=c_{1}, p^{\prime}(a)=c_{2},
$$

where $c_{1}, c_{2}$ are real numbers. Then we obtain the following theorem.

Theorem 4. Let $h, H, c_{1}, c_{2}$ be given real numbers. If in addition $u(a, t)$ is known for all $t$ with $0<t$, then there exists at most one function $p$ with the above properties such that (4.1) - (4.4) are fulfilled.

The proof of this theorem can be given by reduction of the stated problem to an inverse Sturm-Liouville problem (comp. H. P. Linke [11]).

\section{References}

[1] I. Ja. Besnockenko, On the determination of the coefficients in parabolic equations (Russ.), Diff. Uravnenija 10, 24-35 (1974).

[2] G. Chavent, Sur une methode de resolution du probleme inverse dans les equations aux dérives partielles paraboliques, C. R. Acad. Sc. Paris 269, 1135-1138 (1969).

[3] ---, Une méthode de resolution de problème inverse dans les équations aux dérivées partielles, Bull. Acad. Polon. Sc., Série sci. techn. 8, 99-105 (1970).

[4] A. Fasano, Sulla inversione di un classico problema di diffusione del calore, Le Matematiche 16, 50-61 (1971).

[5] W. M. Isakov, Uniqueness theorems for Inverse problems of heat potentials (Russ.), Sibir. Mat. Zurn. 17, 259-272 (1976).

[6] A. D. Iskenderov, Multidimensional inverse problems for linear and quasi-linear parabolic equations (Russ.), Dokl. Akad. Nauk USSR 225, 1005-1008 (1975).

[7] W. T. Ivanov, G. P. Smirnov, F. W. Lubyšev, On local direct and inverse problems for the heat equation (Russ.) Diff. Uravnenija 11, 19-26 (1975).

[8] M. M. Lavrentiev, K. G. Resnizkaja, Uniqueness theorems for some non-inear inverse problems at equations of parabolic type (Russ.) Dokl. Akad. Nauk USSR 203, 531-532 (1973).

[9] --, --, On the uniqueness of an inverse problem in the theory 
of heat conduction (Russ.), Math Problems of Geophysics 6 , 117-121 (1975).

[10] Lavrentiev, Romanov, Vasiliev, Multidimensional inverse problems for differential equations, Springer-Verlag Berlin 1970.

[11] H. P. Linke, ther ein inverses Problem fur die Wärmeleitungsgleichung, Wiss. Z. Techn. Hochsch. Karl-Marx-Stadt 18, 445447 (1976).

[12] K. G. Resnizkaja, An existence and uniqueness theorem for a non-linear one-dimensional-inverse problem of the theory of heat conduction (Russ.), Math. Problems of Geophysics 4, 131134 (1973).

[13] -- Uniqueness of the solution of a non-linear inverse problem of the theory of heat conduction in the analytic case (Russ.), Math. Problems of Geophysics 4, 135-139 (1973).

[14] --, An existence and uniqueness theorem for a non-linear inverse problem of the theory of heat conduction - the method of Newton-Kantorovic (Russ.), Math. Problems of Geophysics 3, 152163 (1972).

[15] V. T. Romanov, Certain inverse problems for equations of hyperbolic type (Russ.), Izdat. "Nauka" Sibirsk. Otdel. Novosibirsk 1972 .

Author's address: Sektion Mathematik Technische Hochschule DDR 90 Karl-Ma.rx-Stadt, PSF 964, G.D.R. 